disease. High monozygotic twin discordance implies a role for non-genetic effects in disease pathogenesis. Previous epigenome-wide association studies (EWAS) have demonstrated a role for DNA methylation in SLE, but different immune cell subsets have so far been insufficiently characterised. The disease discordant twin model is a powerful design for detecting SLE-associated DNA methylation variation.

Objectives: To investigate genome-wide DNA methylation changes in sorted CD4+ T-cells, monocytes, granulocytes and B-cells from twin pairs with at least one SLE-affected twin.

Methods: Altogether 15 SLE twin pairs participated in the study, of which 6 pairs were monozygotic (MZ) and 9 were dizygotic (DZ), including 2 concordant pairs (1 MZ, $1 \mathrm{DZ}$ ). Disease activity was evaluated using the SLE disease activity index (SLEDAI). Peripheral blood was processed using gradient density centrifugation for the granulocyte fraction and the mononuclear cell fraction was sorted serially for CD14+ monocytes, CD4+ T-cells and B-cell enrichment using a RoboSep device (Stemcell Technologies). DNA was extracted using the DNA/RNA/miRNA Universal kit (Qiagen). Genome-wide DNA methylation was evaluated using the Infinium HumanMethylation450K BeadChip (Illumina). Paired analyses were performed using a Wilcoxon test with $p<0.01$ and median differential methylation $>7 \%$ considered as statistically significant. Top differentially methylated genes were validated using pyrosequencing.

Results: In paired analyses of discordant SLE twins without restriction to probe category, we found $176,510,393$ and 2882 differentially methylated CpGs in CD4+ T-cells, monocytes, granulocytes and B-cells, respectively. Restricted to the promoter and transcription start sites, there were 55, 327, 247 and 1628 genes in CD4+ T-cells, monocytes, granulocytes and B-cells with differentially methylated CpGs, respectively. In all cell types, there was a profound hypomethylation of interferon-regulated genes, including IFI44L, DTX3L, PARP9 and IFITM1, which was more pronounced in twins with recent flare within the past 2 years. In contrast to the other cell types, hypermethylation was predominantly observed in B-cells. Using Ingenuity Pathway Analysis, the top upstream regulators of hypermethylated genes in B-cells were TNF, miR-146a-5p and EP300. Hypomethylation of CpGs was validated in all cell types at the genes IFI44L, PARP9, IFITM1, LGALS3BP, LOXL1, MIR1OA, PLSCR1 and RSAD1, in B-cells and granulocytes at TACSTD2 and at MIR146B in B-cells. Hypermethylation of CpGs was validated in B-cells alone for CXCR5, DDR1 and TRAF5.

Conclusions: Robust hypomethylation of interferon-regulated genes is common to all major cellular compartments in SLE twins. The finding of hypermethylated CpGs in B-cells is novel and might be of interest to SLE pathogenesis.

Disclosure of Interest: None declared

DOI: 10.1136/annrheumdis-2017-eular.6090

\section{THU0218 CIRCULATING MICRORNAS AS BIOMARKERS FOR DIAGNOSIS AND TYPIFYING THE ATHEROTHROMBOTIC STATUS IN ANTIPHOSPHOLIPID SYNDROME}

C. Perez-Sanchez ${ }^{1}$, I. Arias-de la Rosa ${ }^{1}$, M.A. Aguirre ${ }^{1}$, P. Ruiz-Limon ${ }^{1}$, N. Barbarroja ${ }^{1}$, Y. Jimenez-Gomez ${ }^{1}$, M.C. Abalos-aguilera ${ }^{1}$,

E. Collantes-Estevez ${ }^{1}$, M. Teresa-Herranz ${ }^{2}$, J. Lozano-Herrero ${ }^{2}$, C. Martinez ${ }^{2}$, R. Gonzalez-Conejero ${ }^{2}$, M.J. Cuadrado ${ }^{3}$, C. Lopez-Pedrera ${ }^{1} .{ }^{1} / M I B I C / R e i n a$ Sofia Hospital/University of Cordoba, Cordoba; ${ }^{2}$ IMIB-Arrixaca /University of Murcial Internal Medicine Service, Morales Meseguer Hospital, Murcia, Spain; ${ }^{3}$ Lupus Research Unit and St. Thomas Hospital, London, United Kingdom

Background: The course of antiphospholipid syndrome (APS) may rapidly progress from asymptomatic to severe manifestations. Thus, timely diagnosis is essential to improve accuracy of therapy. The role of circulating microRNAs (miRNAs) as potential biomarkers of disease has not yet been analysed in APS. Objectives: To investigate the contribution of circulating miRNAs to the pathogenesis of APS and their potential role as non-invasive biomarkers of the disease.

Methods: Ninety APS patients and 42 healthy donors (HD) were included in the study. Clinical and inflammatory parameters were analysed. As a complement to standard clinical follow-up, the ankle-brachial index ( $\mathrm{ABI}$ ) and carotid intima-media thickness (CIMT) were determined. miRNA expression profiling was performed in plasma by PCR-Array, and the Ingenuity Pathways analysis software (IPA) was used to identify specific miRNAs and target proteins associated to the pathogenesis of APS. RT-PCR and ELISA/Bioplex of selected genes and proteins were used to validate microarray data. The diagnostic value of specific miRNAs signatures (ratios) as disease biomarkers was evaluated by ROC curves analysis. To assess the specificity of these signatures in APS, the expression of the selected miRNAs was analysed in plasma from 23 thrombotic patients without associated autoimmune disease. Monocytes isolated from HD and endothelial cells (ECs) were treated in vitro with antiphospholipid antibodies (aPL-lgGs) purified from the serum of APS patients, and the changes promoted on the levels of selected miRNAs and their potential targets were analysed.

Results: The PCR-Array identified 39 circulating miRNAs differentially expressed, including 19 up-regulated and 20 down-regulated in APS. IPA analysis recognized 11 miRNAs as potential modulators of target genes involved in the physiopathology of APS. Logistic Regression and ROC curve analyses identified a signature of 10 miRNA ratios as biomarkers for diagnosis of APS with great accuracy (AUC:0.81), along with 2 miRNA ratios as biomarkers for typifying the atherothrombotic status (pathologic CIMT) of APS (AUC:0.76). Patients with thrombosis but without associated autoimmune disease displayed a specific miRNA profile, distinct from that of APS patients. The miRNA signature was related to clinical features of APS such as the occurrence of foetal loss and the type of thrombosis suffered, and correlated with parameters linked to autoimmunity (aPL-lgG titers), inflammation, and thrombosis (ABI, ESR, TF, PAI-1, MCP-1, VEGF-A and VEGF-R1). In vitro treatment of monocytes and endothelial cells with aPL-IgG antibodies promoted a significant deregulation in the secreted levels of the selected miRNAs and atherothrombotic target proteins.

Conclusions: miRNA levels in the serum of APS patients -modulated by aPL-IgG antibodies- are potential novel biomarkers for diagnosis and typifying of their atherothrombotic status, thus constituting a useful tool in the prevention and management of the disease.

Acknowledgements: Supported by CTS794 and ISCIII (FIS15/1333 and RIER RD16/0012/0015).

Disclosure of Interest: None declared

DOI: 10.1136/annrheumdis-2017-eular.4699

\section{THU0219 AUTOANTIBODY PROFILE OF CHILDREN WITH JUVENILE DERMATOMYOSITIS FROM A TERTIARY CARE CENTRE IN NORTH INDIA}

S. Guleria ${ }^{1,1}$, A. Sharma, A. Gupta, A. Rawat, D. Suri, S. Singh. Pediatric allergy and immunology, Pgimer Chandigarh, Chandigarh, India

Background: Juvenile dermatomyositis (JDM) is a rare childhood autoimmune inflammatory muscle disorder that can result in severe disability or death. Children with JDM can have autoantibodies in their sera like other autoimmune diseases [1]. Over the last few years, few novel Myositis Specific Antibodies (MSA) have been identified. Some phenotypical associations have been described with these autoantibodies like anti p-140 (anti NXP2) has been shown to have correlation with calcinosis in children with JDM [1-2].

Objectives: To study autoantibody profile and to look for phenotypical associations of autoantibodies in JDMS.

Methods: Cross-sectional retrospective study. All children diagnosed to have JDM, registered from 1995 to 2015 in Pediatric Rheumatology Clinic at Post Graduate Institute of Medical Education and Research Chandigarh, India and who were tested for autoantibodies were included in the study. Clinical findings, antinuclear antibodies (ANA), autoantibody for MSA and myositis associated autoantibodies (MAA) were noted from the careful scrutiny of case records. Immunoglobulin G (IgG) antibodies against Jo1, threonyl-tRNA synthetase (PL7), alanyl-tRNA synthetase (PL12), glycyl-tRNA synthetase (EJ), Signal Recognition Particle (SRP), Mi-2, MDA-5, Transcriptional intermediary factor $1-\gamma(\mathrm{TIF}-1 \gamma)$, $\mathrm{Ku}, \mathrm{PMScl} 100$, Scl 70 and SSA/Ro 52 have been done by Immunodot. Evaluation for anti p-140 or Nuclear Matrix Protein (NXP2) and anti 200/100 or 3-Hydroxy-3-Methyglutaryl-Coenzyme (HMG CoA reductase) was done using ELISA.

Results: Antinuclear antibody (ANA) testing was done in 97 patients. Forty six (47.4\%) tested positive. In addition, MSA and MAA were assessed. Anti-SRP antibodies were present in $4(11.4 \%)$ children, anti-MDA5 in $3(8.6 \%)$, anti-Mi2 in $1(2.9 \%)$ and 1 patient tested positive for anti-SSA/Ro52 antibodies. All 4 children with anti-SRP were girls, had polycyclic course and 2 of them developed calcinosis. Patients with anti-MDA5 had predominant skin involvement, less severe muscle disease and followed a monocyclic course. Two of them had arthritis/arthralgia at the time of presentation. The only patient with anti-Mi2 had normal muscle strength/endurance at the time of follow up. None of the patients had anti synthetase antibodies (anti-Jo1, anti-PL-7, anti-PL-12, anti-EJ), anti-ku or anti-Scl-70. None of the subjects tested positive for anti-NXP2 or anti- HMG CoA.

Conclusions: Prevalence of autoantibodies in children with JDM in our study is similar to what has been described previously. Type of autoantibodies, though, is not similar. This may be due to ethnic differences of the population. Autoantibodies were tested in children while they were on treatment. This may have resulted in lower positivity. Evaluation of autoantibody profile at the time of diagnosis may assist in predicting the course of disease and response to treatment.

References:

[1] Targoff IN, Mamyrova G, Trieu EP, Perurena O, Koneru B, O'Hanlon TP, et al. A novel autoantibody to a $155-\mathrm{kd}$ protein is associated with dermatomyositis. Arthritis Rheum 2006; 54: 3682-9.

[2] Gunawardena H, Wedderburn LR, Chinoy H, Betteridge ZE, North J, Ollier WER, et al. Autoantibodies to a $140-\mathrm{kd}$ protein in juvenile dermatomyositis are associated with calcinosis. Arthritis Rheum 2009;60: 1807-14.

Disclosure of Interest: None declared

DOI: 10.1136/annrheumdis-2017-eular.2954

\section{THU0220 SELETALISIB, A NOVEL SELECTIVE PI3K $\Delta$ INHIBITOR WITH THERAPEUTIC POTENTIAL IN INFLAMMATION AND AUTOIMMUNITY}

A. Payne, M. Juarez, G.I. Johnston, E. Helmer, M. Thomson. UCB Pharma, Slough, United Kingdom

Background: PI3K $\delta$ is predominantly expressed in lymphocytes; the role it plays in immune disease has encouraged the development of inhibitors targeting 
its kinase activity. Inhibitors of $\mathrm{PI} 3 \mathrm{~K} \delta$ have been approved or are in clinical development for the treatment of B cell malignancies; their therapeutic potential in inflammatory and autoimmune disease is being explored. Seletalisib is a selective and potent PI3K $\delta$ inhibitor that has been profiled in preclinical and early clinical studies.

Objectives: To assess the therapeutic potential of seletalisib in inflammatory and autoimmune disease.

Methods: In vitro cell-based studies were performed on blood samples taken from healthy volunteers (HV) or from patients with primary Sjogren's Syndrome (pSS) and/or psoriasis. In addition, samples were analysed from a single ascending dose study in HV (NCT02207595) and a multiple ascending dose study in HV and psoriasis patients (NCT02303509). T and B cell responses in the presence and absence of seletalisib were assessed by flow cytometry or a Meso-Scale discovery assay (Meso-Scale Diagnostics, MD, USA), following activation of the T cell- or B cell-receptor by receptor cross-linking or via stimulation with specific antigens. The functional selectivity of seletalisib was assessed in the BioMap system (DiscoverX, CA, USA). Translational studies used flow cytometry and/or immunofluorescence to demonstrate the presence of active PI3K signalling in diseased tissue, through expression of phosphorylated-AKT (pAKT) or -ribosomal protein S6 (pS6). The effect of seletalisib on PI3K signalling was determined by phospho-flow cytometry. Target engagement was determined through measurement of basophil degranulation in healthy subjects, and assessment of lesion severity score in skin biopsies from patients with psoriasis that were treated with seletalisib.

Results: Preclinical studies showed seletalisib potently inhibited T cell differentiation and function ( $\mathrm{IC}_{50}$ range: 2-31 nM). Further, it blocked activation and proliferation of $B$ cells $\left(\mathrm{IC}_{50}\right.$ range: $\left.16-49 \mathrm{nM}\right)$. When profiled in a wide range of primary cell assay systems, including fibroblasts, epithelial, endothelial and vascular smooth muscle cells, seletalisib showed significant activity only in those systems containing lymphocytes, demonstrating its functional selectivity towards PI3K $\delta$-expressing cells. Expression of the PI3K pathway in lymphocytes was shown at the site of disease in clinical samples both from patients with psoriasis and pSS. Seletalisib inhibited PI3K signalling, measured by a reduction in PAKT and pS6 expression, in T cells derived from patients with psoriasis.

In first-in-man studies, mean seletalisib plasma concentration-time profiles increased with increasing dose after single and multiple dosing, with no major deviations from dose proportionality. There was no unexpected accumulation or loss of exposure after multiple dosing (time-independent pharmacokinetic (PK) profile) and apparent $t_{1 / 2}$ values (approx. 20h) were supportive of once-daily dosing. Inhibition of basophil degranulation in healthy subjects and effects on the cellular composition in lesional skin biopsies from patients with psoriasis, provided indications of target engagement, following treatment with seletalisib.

Conclusions: Seletalisib is a potent, selective PI3K $\delta$ inhibitor with an attractive preclinical and human PK profile; clinical studies are ongoing.

Disclosure of Interest: A. Payne Shareholder of: UCB Pharma, Employee of: UCB Pharma, M. Juarez Shareholder of: UCB Pharma, Employee of: UCB Pharma, G. Johnston Shareholder of: UCB Pharma, Employee of: UCB Pharma, E. Helmer Shareholder of: UCB Pharma, Employee of: UCB Pharma, M. Thomson Shareholder of: UCB Pharma, Employee of: UCB Pharma

DOI: 10.1136/annrheumdis-2017-eular.5132

\section{THU0221 DOWNREGULATED EXPRESSION OF MIR200B-5P IN MINOR SALIVARY GLANDS (MSG) OF PATIENTS WITH SJÖGREN'S SYNDROME (SS) ASSOCIATED LYMPHOMA}

E.K. Kapsogeorgou, A. Papageorgiou, M. Voulgarelis, A.G. Tzioufas. Pathophysiology, School of Medicine, National and Kapodistrian University of Athens, ATHENS, Greece

Background: The miRNAs of the miR-200 family are critical regulators of oncogene and tumor suppressor genes. Preliminary data from a limited number of patients with SS-associated lymphoma suggested that the expression of miR200b-5p in MSGs may be downregulated in lymphoma.

Objectives: To validate whether low miR200b-5p MSG-levels are associated with SS-related lymphomas and if they are deregulated before lymphoma development, suggesting a possible prognostic value.

Methods: miR200b-5p expression was analyzed by quantitative real-time PCR in total RNA from MSG tissues obtained from 77 SS patients and 9 patients with non-SS sialadenitis associated with sarcoidosis, HCV infection (4 each) or HBV (1 that was also diagnosed with MALT lymphoma). with chronic sialadenitis associated with sarcoidosis, HCV (4-each) or HBV infection (1 who was also diagnosed with MALT-Iymphoma). SS-patients included 28 that did not develop lymphoma during follow-up (without lymphoma; median follow-up time since biopsy performance, range: 6yrs, $1-12.75 y$ rs), 18 that developed lymphoma in the future (prelymphoma; median follow-up time till lymphoma diagnosis, range: $3.59 \mathrm{yrs}$, 0.42-8.5yrs, 15-MALT, 2-NMZL, 1-DLCBL) and 32 with SS-associated lymphoma at the time of biopsy (lymphoma; 25-MALT, 2-NMZL, 2-DLCBL, 1-BALT, 1-LP and 1 -SLL). In 15 cases, we had sequential MSG-samples from prelymphoma patients who transitioned to lymphoma (12-MALT, 2-NMZL, 1-DLCBL).

Results: Tukey's multiple comparison revealed that miR200b-5p levels were significantly down-regulated in MSG tissues of prelymphoma and lymphoma SSpatients (mean relative expression \pm SE: $0.37 \pm 0.10$ and $0.26 \pm 0.06$, respectively) compared to SS-patients without lymphoma $(0.67 \pm 0.07 ; p \leq 0.05$ and $p \leq 0.0001$ for pre- and lymphoma, respectively) or non-SS sialadenitis $(0.85 \pm 0.28, p \leq 0.05$ and $p<0.01$, respectively). Interestingly, low miR200b-5p levels were detected in HBV patient that had MALT Iymphoma (0.17). The expression of miR200b-5p was not found to differ between patients with SS without lymphoma and nonSS sialadenitis, or SS-associated pre-lymphoma and lymphoma. The analysis of the 15 cases of SS patients that had sequential samples before and on lymphoma diagnosis revealed that miR200b-5p levels do not significantly change over transition to lymphoma. The miR200b-5p expression levels were negatively correlated with the biopsy focus score $(r:-0.6550, p<0.0001)$, whereas they were not associated with the site or the number of involved sites, the type or the stage of lymphoma.

Conclusions: The significantly low levels of miR200b-5p in MSG tissues of patients with SS-associated prelymphomas and lymphomas suggest that miR200b-5p deregulation is implicated in SS-lymphomagenesis. The downregulation of miR200b-5p in prelymphoma samples and the lack of change over transition to lymphoma suggest that it can serve as a prognostic marker for future lymphoma development. The prognostic value of miR200b-5p in SS-associated lymphomas, the expressing cell types and affected molecular pathways are under investigation.

Acknowledgements: Funded by the Hellenic College of Rheumatology

Disclosure of Interest: None declared

DOI: 10.1136/annrheumdis-2017-eular.6708

\section{THU0222 PROPHYLACTIC AND THERAPEUTIC ADMINISTRATION OF AN ANTI-CD40 ANTAGONIST ANTIBODY BLOCKS AND REVERSES PROTEINURIA AND NEPHRITIS IN NZB/W-F1 MICE}

S. Perper, S. Westmoreland, Z. Liu, M. Duval, J. Seagal, B. McRae, S. Clarke. Abbvie, Worcester, United States

Background: The CD40-CD40L pathway is a potential target for the treatment of autoimmune diseases, as CD40 is a costimulatory receptor on antigen presenting cells (APCs) critical for the induction and maintenance of an immune response through binding CD40L on T cells. In the absence of CD40, both humoral and cellular responses to foreign antigens are severely impaired. In particular, disruption of this pathway prevents the development of disease in lupus-prone mice.

Objectives: The objective of this work is to evaluate whether prophylactic and therapeutic treatment with an antagonistic anti-CD40 antibody prevents and reverses nephritis and other manifestations of lupus in lupus-prone mice.

Methods: NZB/W- $F_{1}$ mice were i.p. administered anti-CD40 prophylactically or therapeutically at various doses once or twice per week. Prophylactic treatment of NZB/W- $F_{1}$ mice began at 26 weeks of age and continued for 9 weeks, while therapeutic treatment was initiated in NZB/W- $\mathrm{F}_{1}$ mice after they developed severe proteinuria (urine protein $>300 \mathrm{mg} / \mathrm{dL}$ ). Proteinuria was monitored weekly by urinalysis, and at study termination blood and spleen cells were analyzed by flow cytometry. Additionally, histological analysis of kidney, spleen, and salivary glands were performed, as well as gene transcription analysis of the kidney by microarray.

Results: As expected, prophylactic and therapeutic administration reduced the number of splenic germinal center (GC) B cells and T follicular helper cells (Tfh). Prophylactic treatment blocked development of proteinuria and extended survival in NZB/W- $F_{1}$ mice. Significantly, therapeutic anti-CD40 treatment reversed established, severe proteinuria in NZB/W- $\mathrm{F}_{1}$ mice and extended their survival. In agreement, the kidneys and salivary glands of treated mice exhibited reduced inflammation compared to control mice, and the kidneys exhibited lower CD40, IFN-I and chemokine gene signatures compared to controls. Interestingly, antiCD40 decreased expression of genes in kidney belonging to the "hepatic fibrosis" pathway, possibly explaining the improvement of kidney function.

Conclusions: Anti-CD40 treatment prevents the onset and reverses ongoing nephritis and sialadenitis in NZB/W- $\mathrm{F}_{1}$ mice. Resolution of ongoing nephritis results in restoration of low or normal urine protein levels. Thus, treatment with an antagonistic anti-CD40 is a strong candidate for clinical study in SLE.

Disclosure of Interest: S. Perper Employee of: Abbvie, S. Westmoreland Employee of: Abbvie, Z. Liu Employee of: Abbvie, M. Duval Employee of: Abbvie, J. Seagal Employee of: Abbvie, B. McRae Employee of: Abbvie, S. Clarke Employee of: Abbvie

DOI: 10.1136/annrheumdis-2017-eular.1583

\section{THU0223 ELEVATED MTORC1 SIGNATURE IN B CELLS FROM SJÖGREN'S SYNDROME PATIENTS CORRELATES WITH B CELL HYPERACTIVITY THAT IS ABROGATED BY MTOR INHIBITION: A NOVEL THERAPEUTIC STRATEGY TO HALT B CELL HYPERACTIVITY IN PSS?}

S.L. Blokland ${ }^{1,2}$, M.R. Hillen ${ }^{1,2}$, C.G. Kommer-Wichers ${ }^{1,2}$, A.A. Kruize ${ }^{1}$, J.C. Broen ${ }^{1,2}$, J.A. van Roon ${ }^{1,2}$, T.R. Radstake ${ }^{1,2} .{ }^{1}$ Rheumatology \& Clinical Immunology; ${ }^{2}$ Laboratory of Translational Immunology, UMC Utrecht, Utrecht, Netherlands

Background: A hallmark feature of primary Sjögren's syndrome (pSS) is B cell hyperactivity, including presence of autoantibodies, aberrant presence of $B$ cells and plasma cells in the salivary glands, elevated serum IgG levels and increased risk of lymphoma development. The mTOR pathway is essential for cell growth, 